\title{
Patient Outcome in Pregnancy Requiring Dialysis: A Case Series
}

\author{
Karina Sulaiman", Madhavilatha Vuppali and Kenneth Abreo
}

Department of Medicine, Nephrology Section, Louisiana State University Health Sciences Center, Shreveport, Louisiana

\begin{abstract}
The optimal management of pregnant dialysis patients remains a great challenge for nephrologists, end-stage renal disease being a predictor of adverse outcomes in this condition. We report a single-center experience of four patients requiring dialysis during pregnancy, all of which resulted in successful delivery of viable infants. Our success rate may reflect an overall improvement in management of this population, with special attention paid to multiple risk factors. These include blood pressure and volume control, anemia management with erythropoietin analogues, nutritional intake and total dose of dialysis.
\end{abstract}

Keywords: Hemodialysis, outcomes, pregnancy.

\section{INTRODUCTION}

Women with end-stage renal disease (ESRD) who are on dialysis rarely get pregnant and if they do, pregnancy outcomes are fraught with risks to infant and mother. Advanced chronic kidney disease leads to secondary amenorrhea from anovulation and uremia diminishes the luteal hormone surge and estradiol peak essential for conception [1]. Reported conception rates have been exceedingly low worldwide [2-4], with infant survival rates dropping if conception occurred after dialysis initiation [5].

Once pregnancy occurs in patients with chronic kidney disease unique management issues come into play and a thorough knowledge of these helps the nephrologist and obstetrician navigate the patient to a successful pregnancy. Since the publication of the first successful pregnancy in a dialysis patient in 1971 [6], the optimal management of pregnant dialysis patients has been an area of much discussion. The nature of this condition and the limited numbers preclude the inclusion of these patients in large clinical trials. Hence the bulk of data available to clinicians to help guide management is largely in the form of case reports and series. Despite major advances in this field, chronic and end-stage renal disease remains a major predictor of adverse outcomes in pregnancy [7]. In the $1990 \mathrm{~s}$, a rate of $30 \%$ for first-trimester and $15 \%$ for second-trimester loss were reported. These studies also reported a live birth rate of $52 \%$ and an infant survival rate of $37 \%$ [8].

Herein, we report a single-center experience from 2003 to 2010, of four cases of pregnant patients who had ESRD requiring dialysis. Each of these cases resulted in the delivery of a live fetus. This improved outcome may reflect an overall improvement in management of this population, with special attention paid to multiple risk factors. These include blood pressure, intravascular volume, anemia, acid-base balance, nutrition and adequacy of dialysis.

*Address correspondence to this author at the Section of Nephrology, LSU Health Sciences Center, 1501 Kings Highway, Shreveport, LA 71130, USA; Tel: 318-675-7402; Fax: 318-675-5913; E-mail: ksulai@1suhsc.edu

\section{CASE REPORTS}

\section{Case 1}

A 39-year-old African American female G8, P1-1-6 was admitted to the LSU Health Sciences Center at 25 weeks of gestational age. Her past medical history included hypertension, diabetes mellitus, and hepatitis B and C. She also had a history of chronic kidney disease (CKD stage 4) thought to be secondary to a combination of diabetic nephropathy and extensive cocaine use. She was admitted for management of worsening renal function and uncontrolled hypertension. BUN and creatinine levels on admission were $53 \mathrm{mg} / \mathrm{dl}$ and $4.6 \mathrm{mg} / \mathrm{dl}$ respectively (increased from $45 \mathrm{mg} / \mathrm{dl}$ and $2.8 \mathrm{mg} / \mathrm{dl}$ a month prior). Hemodialysis was initiated five days later via a right internal jugular vein tunneled catheter for worsening renal function, hypertension and volume overload. Her blood pressure remained reasonably controlled with ultrafiltration on dialysis and medications. Antihypertensive medications were labetalol $600 \mathrm{mg}$ BID and long-acting nifedipine $90 \mathrm{mg}$ BID in conjunction with hydralazine when needed. In addition, she received phosphate binders, vitamin $\mathrm{D}$, multivitamin supplements and erythropoeitin during dialysis. Her hemoglobin levels rose steadily from $8.5 \mathrm{~g} / \mathrm{dl}$ to within normal range. She underwent dialysis for four hours six days a week until delivery, with appropriate increases being made to her dry weight in consultation with her obstetricians to help estimate fetal growth. The average BUN and creatinine on dialysis were $31 \mathrm{mg} / \mathrm{dl}$ and $3.0 \mathrm{mg} / \mathrm{dl}$. She successfully delivered a baby girl by Caesarean section at 34 weeks, with APGARS 9 and 9. Dialysis was continued from the day of delivery until her discharge and she remains dialysis dependent to this day. The child is healthy and developing normally as reported by the patient.

\section{Case 2}

A 34-year-old African American woman with a history of diabetes mellitus with proteinuria and retinopathy, hypertension and chronic kidney disease was admitted to the 
hospital with a gastrointestinal bleed, urinary tract infection, and deteriorating renal function. She was at twenty two weeks gestational age. Her obstetric history included two preterm caesarian sections at 32 and 30 weeks for nonreassuring fetal heart tones and preeclampsia respectively, and three 1st trimester spontaneous abortions. She also admitted to cocaine use during this pregnancy. Her admission laboratory tests showed: BUN $34 \mathrm{mg} / \mathrm{dl}$, creatinine $4.3 \mathrm{mg} / \mathrm{dl}$, sodium $139 \mathrm{mEq} / \mathrm{L}$, chloride $111 \mathrm{mEq} / \mathrm{L}$, potassium $3.3 \mathrm{mEq} / \mathrm{L}$, bicarbonate $16 \mathrm{mEq} / \mathrm{L}$, and hemoglobin $9.2 \mathrm{~g} / \mathrm{dl}$. Her kidney function deteriorated subsequently, a tunneled catheter was placed in the right internal jugular vein and hemodialysis was initiated. Renal replacement therapy was continued for six days a week, each session lasting 4 hours, until delivery which was a month later at twenty seven weeks gestational age. Her average serum BUN while on dialytic therapy was less than $30 \mathrm{mg} / \mathrm{dl}$. This was attributed to her severe hypoalbuminemia of less than $2 \mathrm{mg} / \mathrm{dl}$, presumably due to diabetic nephropathy with 8 grams of proteinuria and possibly low protein intake. Her course on dialysis was uneventful except for intermittent difficulty controlling her blood pressure which improved after increase in the dose of her long-acting nifedipine to $60 \mathrm{mg}$ PO BID, in addition to methyldopa $250 \mathrm{mg}$ PO TID. She required blood transfusion for her gastrointestinal bleed and later received 3000 units of erythropoietin during dialysis. She was not prescribed any binders during her admission. She delivered a viable male infant with an APGAR score of 6 and 9 by emergency caesarian section following a $40 \%$ placental abruption. She was lost to follow up after the discharge, and information regarding her child's health is not available.

\section{Case 3}

A 38-year-old African American female with a history of chronic kidney disease (CKD stage 5) and uncontrolled hypertension, was admitted to the hospital for blood pressure control at seven weeks gestation. She had four previous pregnancies with two successful deliveries and two miscarriages. She was not on any medications and had a history of marijuana use. Her laboratory tests showed a BUN of $24 \mathrm{mg} / \mathrm{dl}$, creatinine $5.0 \mathrm{mg} / \mathrm{dl}$ and bicarbonate $14 \mathrm{mEq} / \mathrm{L}$, all other electrolytes were within normal limits. A tunneled catheter was placed in the right internal jugular vein and she was initiated on hemodialysis the following day for blood pressure elevation and symptoms of uremia. Hemodialysis was provided 6 days a week with each session lasting 4 hours. She was however noncompliant with both medical therapy and dialysis, signing out against medical advice and refusing admission on several occasions. As such her blood pressure control was extremely variable, but almost always normalized on dialysis. Average BUN and creatinine levels were $25 \mathrm{mg} / \mathrm{dl}$ and $4.5 \mathrm{mg} / \mathrm{dl}$, but renal function did deteriorate towards the date of delivery. She was readmitted later that month at nine weeks gestation on two occasions for episodes of hypertensive urgency with possible seizures. In the subsequent months she underwent hemodialysis at the labor unit six days a week but refused admission multiple times for further monitoring. During the days immediately preceding delivery she was on methyldopa $250 \mathrm{mg}$ TID and labetalol $300 \mathrm{mg}$ BID for blood pressure, and oral iron pills for anemia. She did receive 17,200 units of erythropoietin with her last dialysis session prior to delivery. She underwent emergency caesarian section at thirty three weeks gestation for non-reassuring fetal heart tones and premature rupture of membranes, delivering a viable male infant with an APGAR score of 0, 4 and 7. Her postoperative course was complicated by group B streptococcal bacteremia, for which she received antibiotics. Both mother and child were otherwise healthy. She continued to remain in ESRD and changed her dialysis modality to peritoneal dialysis. Her child is three years old and is in good health.

\section{Case 4}

A 39-year-old African American female G3 P1-0-11 presented at 28 weeks gestation for a dental abscess. Her medical history was significant for diabetes with retinopathy and proteinuria with CKD III/IV, hypertension, mitral valve repair, hyperthyroidism, and atrial fibrillation with a history of cardioversion. Laboratory tests on admission were BUN $31 \mathrm{mg} / \mathrm{dl}$, creatinine $3.2 \mathrm{mg} / \mathrm{dl}$, sodium $132 \mathrm{mEq} / \mathrm{L}$, potassium $3.9 \mathrm{mEq} / \mathrm{L}$, chloride $105 \mathrm{mEq} / \mathrm{L}$, bicarbonate $17 \mathrm{mEq} / \mathrm{L}$, glucose $395 \mathrm{mg} / \mathrm{dl}$, LDH $668 \mathrm{u} / \mathrm{L}$, uric acid $9.6 \mathrm{mg} / \mathrm{dl}$, ALT $10 \mathrm{u} / \mathrm{L}$, AST $23 \mathrm{u} / \mathrm{L}$, calcium $7.8 \mathrm{mg} / \mathrm{dl}$, albumin $2.3 \mathrm{gm} / \mathrm{dl}$ and BNP 15,955 $\mathrm{pg} / \mathrm{ml}$. Hematology revealed anemia and thrombocytopenia. As her liver function tests remained normal she was not thought to have HELLP syndrome. Her abscess was drained; she was started on antibiotics but left against medical advice without being dialyzed. She was readmitted two days later with progressive dyspnea, leg swelling and worsening renal function. An internal jugular tunneled catheter was placed and hemodialysis initiated. She underwent daily dialysis, with appropriate increases being made to her dry weight in consultation with her obstetricians. The average BUN ranged between 30 and $40 \mathrm{mg} / \mathrm{dl}$. She was given intravenous iron for anemia and did not receive any erythropoeitin. Her hemoglobin was maintained around $10 \mathrm{~g} / \mathrm{dl}$ throughout her pregnancy. Her blood pressure remained elevated (average BP 140/110) except during dialysis despite adjustment in ultrafiltration on dialysis and treatment with carvedilol, longacting nifedipine and hydralazine. At 29 weeks, she underwent emergent caesarian section due to persistently elevated blood pressure, thrombocytopenia and abnormal liver function tests. She delivered a viable infant with an APGAR score of 8 and 9. Until last follow up the child was alive and healthy. She remained dialysis dependent thereafter.

\section{DISCUSSION}

Pregnancy in advanced kidney disease has traditionally been thought to be associated with poor outcomes, with early studies reporting live birth rates anywhere between $9-23 \%$ $[2,9]$. Babies born were likely to be premature, further decreasing the likelihood of progressing to healthy lives. Women on dialysis were thus previously counseled to avoid pregnancy. Recent studies have reported more successful outcomes based on observational data, which include different modalities of renal replacement therapy [10]. However, if pregnancy were to occur in a patient on hemodialysis today, the results reported in the literature [11] and our single center 
experience $(80 \%$ fetal survival due to one pregnancy resulting in neonatal demise shortly after delivery) would suggest a favorable outcome for pregnancy. In a recent case series reported by Luders et al. from Brazil the success rate was $87 \%$ and was attributed to advances in care of the pregnant dialysis patient [12].

A recurrent theme in the success of pregnancy in hemodialysis appears to be the increased and sustained dialysis dosing throughout gestation. This theory has been suggested since the 1980s [9], but not until recently has significant increases in dialysis dosing led to reported deliveries of viable infants $[3,13]$. Centers are now developing protocols based on factors affecting pregnancy outcomes to standardize the care of this population [14]. The increased frequency and dosage of hemodialysis leads to lower blood urea concentration and better control of volume and blood pressure. In addition to ultrafiltration of fluid gained during the interdialytic interval, adjustments to the dry weight of the patient must be made, to accommodate the growing fetus. In the second and third trimester the dry weight should be increased by $0.5 \mathrm{~kg}$ each week. Excessive ultrafiltration should be avoided as extreme falls in blood pressure during hemodialysis will compromise fetal blood flow. The dialysis regimen in each of our patients involved dialysis 6 days each week achieving a total dose in excess of 20 hours per week that resulted in a blood urea nitrogen level less than $50 \mathrm{mg} / \mathrm{dl}$ and optimal blood pressure control (Tables 1 and 2). Daily dialysis can be provided by two other modalities, namely nocturnal hemodialysis and peritoneal dialysis. The use of nocturnal hemodialysis has been reported to result in successful outcomes in pregnancy, with the most promising results being reported in five women who had seven pregnancies and delivered six live infants using this modality [15]. Several centers have reported successful pregnancies with peritoneal dialysis [16]; however registry data do not show differences in outcome between hemodialysis and peritoneal dialysis [5].

Besides providing adequate hemodialysis there are several other complications of ESRD that have to be addressed in the pregnant hemodialysis patient. Hypertension is a commonly reported maternal complication $[11,17]$, and was present in each of our patients (Table 1). Commensurate with our experience, adequate control of blood pressure can decrease maternal complications and increase the likelihood of a successful delivery. Since ultrafiltration alone was insufficient to control the blood pressure, combinations of antihypertensive medications, known to be safe in pregnancy, were administered. These medications included long-acting nifedipine, methyldopa, hydralazine, and carvedilol.
Iron deficiency anemia is common in pregnant women especially in the third trimester as fetal demands for iron increase [18]. It has been shown to increase preterm delivery and subsequent low birth weight [18]. Besides pregnancyrelated factors, chronic renal disease and the hemodialysis procedure are additional risk factors for iron deficiency. Since chronic kidney disease is an inflammatory state, increased levels of the protein hepcidin interfere with the gut absorption of iron and the hemodialysis procedure itself results in blood loss in the dialyzer. Thus, the nephrologist caring for the pregnant hemodialysis patient should frequently check iron stores and provide intravenous iron to prevent iron-deficiency anemia. Anemia also results from lack of erythropoietin which can be easily administered either IV or SQ when the patient is receiving hemodialysis. Erythropoietin can be safely administered during pregnancy and is well tolerated. It does not appear to cross the placenta due to its large size and there has been no reported fetal or maternal morbidity from its use [19]. Cytokine production in gestation may lead to erythropoietin resistance [3]; despite receiving erythropoietin (Table 1), only 3 patients were within the goal of 10-11 g/dl prior to delivery (Table 2).

Table 1. Summary of demographics, dialysis time, blood pressure control, anemia and fetal outcome.

\begin{tabular}{|c|c|c|c|c|}
\hline Case & $\mathbf{1}$ & $\mathbf{2}$ & $\mathbf{3}$ & $\mathbf{4}$ \\
\hline \hline Age (yrs) & 39 & 34 & 38 & 39 \\
\hline Race & AA & AA & AA & AA \\
\hline HD (hr) & 24 & 24 & 24 & 24 \\
\hline HTN & Controlled & Controlled & Variable & Variable \\
\hline EPO & Yes & Yes & Yes & No \\
\hline GA* & 34 & 27 & 33 & 29 \\
\hline Mode & C-section & C-section & C-section & C-section \\
\hline BW (g) & 1580 & 730 & 1877 & 1340 \\
\hline APGAR & $9 / 9$ & $6 / 9$ & $0 / 4 / 7$ & $8 / 9$ \\
\hline
\end{tabular}

$\mathrm{GA}^{*}=$ gestational age in weeks, $\mathrm{BW}=$ fetal weight at birth, Mode = type of delivery. HD (hr/week).

Pregnant women have a chronic respiratory alkalosis from stimulation of the respiratory center by progesterone and lifting of the diaphragm by the enlarged uterus. The serum bicarbonate is typically $20-22 \mathrm{mg} / \mathrm{dl}$ in compensation for the respiratory alkalosis. The pregnant hemodialysis patient should be dialyzed against a lower bath concentration of bicarbonate to achieve lower serum bicarbonate levels $[14,16]$. Hypophosphatemia can develop in patients due to

Table 2. Laboratory data on all patients drawn on date of last dialysis prior to delivery.

\begin{tabular}{|c|c|c|c|c|c|c|c|}
\hline Pt & BUN (mg/dl) & Cr (mg/dl) & HCO3 (mmol/L) & K (mmol/L) & Ca (mg/dl) & Phos (mg/dl) & Albumin (g/dl) \\
\hline \hline 1 & 41 & 4.2 & 22 & 4.3 & 8.9 & 5.7 \\
\hline 2 & 11 & 2.6 & 25 & 3.9 & 7.9 & 3.5 & 11.3 \\
\hline 3 & 16 & 3.9 & 28 & 5.1 & 9.5 & 6.0 \\
\hline 4 & 50 & 4.8 & 18 & 4.4 & 8.4 & $4.3 *$ \\
\hline
\end{tabular}

*Serum phosphorus was drawn 2 days prior to delivery. 
daily dialysis especially in patients who are compliant with a low phosphorus diet and phosphate binders. Two of the four patients in this series had phosphate levels above the goal of $5.5 \mathrm{mg} / \mathrm{dl}$ just prior to delivery. All patients received folate and prenatal vitamins as water soluble vitamins are lost across the dialysis membrane.

Premature delivery in these patients continues to be an issue, as confirmed by the cases presented, and progress in the quality of care delivered to these neonates has also helped to improve outcomes. Our single-center experience is comparable to similar reports worldwide, and indicates a trend toward improved outcomes in pregnancy, if managed with vigilance and close follow-up. The management of renal disease in the patients at our center was commensurate with proposed targets in the literature. The experience at our center does highlight the challenges of managing high-risk patients with great difficulties in achieving compliance as well as a history of toxic ingestion. Despite these presumably poor prognostic indicators, each pregnancy did result in a live birth and favorable maternal outcomes. Often we were not able to reach the optimal goals recommended due to patient factors. Further study of specific strategies and their relative importance in optimizing care should be further researched. Registry data or pooled information from case series is probably the best approach to develop protocols and guidelines to optimize the care of pregnant patients on hemodialysis.

All four patients became pregnant when they had CKD and were not on hemodialysis. This may have played a role in their ability to conceive and the successful outcome of their pregnancies. Hemodialysis was needed during the course of their pregnancy. All patients came from a poor socio-economic background and therefore did not get prenatal care. In addition they had a history of drug use and were all over thirty years of age. However, once they needed hemodialysis they received sustained medical attention from their nephrologists and high-risk obstetricians that may have also played a part in the successful outcome of their pregnancies.

\section{CONFLICT OF INTEREST}

The authors confirm that this article content has no conflict of interest.

\section{ACKNOWLEDGEMENTS}

Declared none.

\section{REFERENCES}

[1] Palmer BF. Sexual dysfunction in uremia. J Am Soc Nephrol 1999; 10: $1381-8$.

[2] Toma H, Tanabe K, Tokumoto T, Kobavashi C, Yagisawa T. Pregnancy in women receiving renal dialysis or transplantation in Japan: a nationwide survey. Nephrol Dial Transplant 1999; 14: 1511-6.

[3] Bagon JA, Vernaeve H, De Muylder X, Lafontaine JJ, Martens J, Van Roost G. Pregnancy and dialysis. Am J Kidney Dis 1998; 31: 756-65.

[4] Souqiyyeh MZ, Horaib SO, Saleh AG, Aswad G. Pregnancy in chronic hemodialysis patients in the Kingdom of Saudi Arabia. Am J Kidney Dis 1992; 19: 235-38.

[5] Okundaye I, Abrinko P, Hou S. Registry of pregnancy in dialysis patients. Am J Kidney Dis 1998; 31: 766.

[6] Confortoni P, Galanti G, Ancona G, Giongio A, Bruschi E, Lorenzo E. Full term pregnancy and successful delivery in a patient on chronic hemodialysis. Proc Eur Dial Tranplant Assoc 1971; (8): 74-80.

[7] Piccoli GB, Attini R, Vasario E, et al. Pregnancy and chronic kidney disease: a challenge in all CKD stages. Clin J Am Soc Nephrol 2010; 5: 844-55.

[8] Chan WS, Okun N, Kjellstrand CM. Pregnancy in chronicdialysis: a review and analysis of the literature. Int J Artif Organs 1998; 21(5): 259-68.

[9] Registration Committee of the European Dialysis and Transplant Association. Successful pregnancies in women treated by dialysis and kidney transplantation. Br J Obstet Gynaecol 1980; 87: 839-45.

[10] Shahir AK, Briggs N, Katsoulis J, Levidiotis V. An observational outcomes study from 1966-2008, examining pregnancy and neonatal outcomes from dialysed women using data from the ANZDATA Registry. Nephrology (Carlton) 2013; 18(4): 276-84.

[11] Yang LY, Thia EWH, Than LK, et al. Obstetric outcomes in women with end-stage renal disease on chronic dialysis: a review Obstet Med 2010; 3: 48-53.

[12] Luders C, Castro MC, Titan SM, et al. Obstetric outcome in pregnant women on long-term dialysis: a case series. Am J Kidney Dis 2010; 56(1): 77-85.

[13] Haase M, Morgera S, Bamberg C, et al. A systematic approach to managing pregnant dialysis patients - the importance of an intensified haemodiafiltration protocol. Nephrol Dial Transplant 2005; 20: 2537-42.

[14] Hladunewich M1, Hercz AE, Keunen J, Chan C, Pierratos A. Pregnancy in end stage renal disease. Semin Dial 2011; 24(6): 634-9.

[15] Barua M, Hladunewich M, Keunen J, et al. Successful pregnancies on nocturnal home hemodialysis. Clin J Am Soc Nephrol 2008; 3: 392-6.

[16] Hou S. Pregnancy in women on haemodialysis and peritoneal dialysis. Baillieres Clin Obstet Gynaecol 1994; 8: 481.

[17] Schneider KT. Pregnancy-induced hypertension: maternal and fetal hemodynamics. Arch Gynecol Obstet 1989; 245: 240-4.

[18] Allen LH. Anemia and iron deficiency: effects on pregnancy outcomes. Am J Clin Nutrition 71, 2000; 1280-4.

[19] Yankowitz J, Piraino B, Laifer SA, et al. Erythropoietin in pregnancies complicated by severe anemia of renal failure. Obstet Gynaecol 1992; 80(3 Pt 2): 485-8. 